\title{
Biorremediação de solos contaminados com resíduos oleosos através de bioaumentação e atenuação natural
}

\section{Bioremediation of Contaminated Soil with Oils Residuals through Bioaugmentation and Natural Attenuation}

\author{
Maitê Carla Deon ${ }^{1}$; Andréia De Rossi²; Clinei Dal'Magro ${ }^{3}$; Christian Oliveira \\ Reinehr ${ }^{4}$; Luciane Maria Colla ${ }^{5}$
}

\section{Resumo}

O potencial de contaminação de solos por vazamentos de óleos vem crescendo, devido à forte industrialização e o desenvolvimento econômico dos países. Diante desse cenário, a biorremediação tem se mostrado uma alternativa para remediar áreas mediante uso de agentes biológicos. Dois microorganismos, isolados de um efluente rico em lipídios, foram utilizadas nos ensaios de bioaumentação em solos contaminados com óleo diesel, óleo lubrificante e óleo de soja. Ensaios de atenuação natural foram realizados como controles. As remoções do óleo diesel no tempo de $21 \mathrm{~d}$ foram de $18,5 \%, 7,30 \%$ e $11,38 \%$ respectivamente, para as técnicas de bioaumento com os isolados I1 e I2 e atenuação natural. As remoções do óleo lubrificante foram de $41,6 \%, 14,16 \%$ e $6,91 \%$ respectivamente, para as técnicas de bioaumento com os isolados I1 e I2 e atenuação natural, enquanto que para o óleo de soja as remoções foram de $87,8 \%, 73,9 \%$ e $49,4 \%$. Considerando-se as técnicas de bioaumento e atenuação natural, o bioaumento com o isolado I1 apresentou melhores resultados, possivelmente devido à produção de compostos com capacidade de diminuição da tensão superficial durante a fase de preparo do bioaumento. Palavras-chave: Óleo Diesel. Óleo lubrificante. Óleo de soja. Efluente de laticínios. Isolamento de micro-organismos para biorremediação.

\begin{abstract}
The potential for soil contamination by oil spills is growing, due to heavy industrialization and economic development of countries. Due to this fact, the bioremediation has become an alternative to remediate areas through the use of biological agents. Two microorganisms, isolated from a lipid-rich effluent, were used in the bioaugmentation of soils contaminated with diesel oil, lubricating oil and soybean oil. Natural attenuation tests were conducted as controls. The removal of diesel fuel at the time of $21 \mathrm{~d}$ were of $18.5 \%, 7.30 \%$ and $11.38 \%$, respectively, for the bioaugmentation with isolated I1 and I2 and natural attenuation. The removal of lubricating oil were $41.6 \%, 14.16 \%$ and $6.91 \%$ respectively for the
\end{abstract}

\footnotetext{
${ }^{1}$ Acadêmicas do Curso de Engenharia Ambiental, Universidade de Passo Fundo; maitcarla@gmail.com,

2 Acadêmicas do Curso de Engenharia Ambiental, Universidade de Passo Fundo; deia_derossi@hotmail.com

${ }^{3}$ Mestrando do Programa de Pós-Graduação em Engenharia, Universidade de Passo Fundo; clineidalmagro@gmail.com

${ }^{4}$ Docente do Curso de Engenharia Ambiental, Universidade de Passo Fundo; reinehr@upf.br

${ }^{5}$ Docente do Programa de Pós-Graduação em Engenharia, Universidade de Passo Fundo; *lmcolla@upf.br
} 
bioaugmentation with the isolated I1 and I2 and natural attenuation, while for soybean oil removals were of $878 \%, 73.9 \%$ and $49.4 \%$. Considering the processes of bioaugmentatiom and natural attenuation, the bioaugmentation with the isolated I1 showed better results, possibly due to the production of compounds capable of reducing the surface tension during the preparation of bioaugmentation.

Key words: Diesel oil. Lubrificating oil. Soybean oil. Dairy effluent. Isolating microorganisms for bioremediation.

\section{Introdução}

As atividades de extração, transporte e refinamento de petróleo têm contribuído para a contaminação do solo com hidrocarbonetos de petróleo em todo o planeta (LOPES; PIEDADE, 2010). Diante disso, observam-se com muita preocupação, os casos de contaminação do solo e água por hidrocarbonetos derivados de petróleo, que mesmo em pequenas concentrações podem constituir um grande perigo à saúde humana e ao meio ambiente (SOUZA et al., 2010).

A contaminação dos solos por vazamentos de óleos como o diesel, de soja e lubrificante podem acontecer de várias maneiras. A contaminação pelo óleo diesel pode ocorrer devido principalmente por vazamentos de tanques de armazenamento subterrâneos em postos de combustíveis, acidentes envolvendo veículos transportadores e falhas mecânicas ou humanas nas operações de descarga (DIEMER et al., 2010). A contaminação pelo óleo de soja ocorre devido ao hábito do descarte incorreto em pias ou diretamente no solo. Já a contaminação por óleo lubrificante é um fato comum devido ao descarte incorreto e vazamentos, principalmente em oficinas mecânicas, e por esses serem mais recalcitrantes, promovem um grande impacto ao meio ambiente em relação a outros tipos de óleos (MACIEL; TAKAKI; GUSMÂO, 2010). Diante disso surge à necessidade de remediação dos ambientes contaminados, o que tem estimulado o surgimento de técnicas de remediação que visam à eliminação desses poluentes (BAPTISTA; CAMMAROTA; FREIRE, 2003). Dentre as técnicas utilizadas, a biorremediação tem se destacado por ser uma tecnologia limpa, de baixo custo e que utiliza a capacidade fisiológica que alguns organismos apresentam em degradar compostos (MACIEL; TAKAKI; GUSMÃO, 2010). A biorremediação utiliza para a descontaminação dos locais micro-organismos autóctones ou alóctones, podendo ser realizada através de bioaumentação, bioestimulação ou atenuação natural monitorada (ANDRADE; AUGUSTO; JARDIM, 2010). A técnica de bioaumentação consiste na introdução de micro-organismos cultivados para degradar cadeias de hidrocarbonetos dentro de um sistema natural contaminado (BARROS; LEMOS, 2006). O processo de bioestimulação consiste em introduzir nutrientes adicionais na forma de fertilizantes orgânicos e/ou inorgânicos na área contaminada, incentivando o crescimento de microorganismos capazes de degradar os poluentes existentes no meio (MILLER, 2010) e, com isso, promovendo o aumento da população microbiana e conseqüentemente, uma degradação mais rápida do contaminante (KANISSERY; SIMS, 2011). $\mathrm{Na}$ atenuação natural monitorada, a degradação do poluente orgânico presente no solo ocorre sem adequação de qualquer condição ambiental, onde a desestruturação do poluente é realizada pelos micro-organismos nativos do local, devido à adaptação natural destes à presença do contaminante (PERELO, 2010). Esses micro-organismos passam, então, a utilizar o composto orgânico poluente como fonte de carbono, ocasionando assim uma redução da sua concentração ao longo do tempo. Nesse sistema não só os processos biológicos estão envolvidos, mas também, processos físicos e químicos podem ser responsáveis pela redução da concentração do poluente (BEZERRA, 2009). 
A biorremediação de solos contaminados com resíduos oleosos depende da capacidade de assimilação desses compostos pelos microorganismos (WETLER-TONINI; REZENDE; GRATIVOL, 2010). Muitos micro-organismos crescem na presença de contaminantes oleosos produzindo biossurfactantes (VIRAMONTESRAMOS et al., 2010). Os biossurfactantes são agentes ativos de superfície que reduzem a energia livre do sistema por substituir a maior parte das moléculas de alta energia na interface diminuindo, assim, a tensão superficial e interfacial dos líquidos, que agem como emulsificantes (BUENO; SILVA; GARCIA-CRUZ, 2010), sendo estes constituídos por uma porção hidrofílica e outra hidrofóbica (COSTA et al., 2010). Essa propriedade aumenta a solubilidade e a disponibilidade de poluentes hidrofóbicos aos microorganismos, aumentando o potencial de biodegradação (COSTA et al., 2010). Os biossurfactantes possuem características benéficas ao meio ambiente e ocorrem naturalmente no solo, além disso, possuem aplicações potenciais em diferentes setores industriais como formulações farmacêuticas e cosméticas, petroquímica, médica, produtos alimentícios e nas áreas de proteção ao meio ambiente (BUENO; SILVA; GARCIACRUZ, 2010). Diversos tipos microbianos são capazes de produzir biossurfactantes, tais como bactérias (PINTO; MARTINS; COSTA, 2009; BUENO, 2008), leveduras (FONTES; AMARAL; COELHO, 2008) e fungos filamentosos (COLLA et al., 2010; MARTINS; KALIL; COSTA, 2008), sendo estes compostos importantes nos processos de biorremediação de resíduos oleosos (NITSCHKE; PASTORE, 2002).

Objetivou-se a biorremediação de solos contaminados por resíduos oleosos através de atenuação natural e bioaumentação por microorganismos isolados de efluentes rico em lipídios.

\section{Material e Métodos}

Amostras de solo

O solo foi coletado no Centro Tecnológico (CETEC) da Universidade de Passo Fundo (UPF), localizada na cidade de Passo Fundo, RS. Segundo a Empresa Brasileira de Pesquisa Agropecuária (Embrapa, 2004), esse solo é classificado como Latossolo Vermelho Distrófico Típico, possuindo $\mathrm{pH}$ em torno de 5,4.

\section{Contaminação do solo e delineamento experimental}

A contaminação do solo foi realizada com óleo diesel, óleo lubrificante e óleo de soja, a fim de simular possíveis contaminações do solo com resíduos oleosos. Os ensaios foram realizados utilizando-se parcelas de solo de $500 \mathrm{~g}$, as quais foram contaminadas com $50 \mathrm{~mL}(10 \%)$ de contaminante e tratadas por atenuação natural e bioaumentação. Os experimentos foram realizados em duplicata, durante $21 \mathrm{~d}$. A umidade foi ajustada semanalmente até $60 \%$ com adição de água e a aeração foi promovida pela mistura manual do solo com auxílio de espátula. Foram coletadas alíquotas de solo para a determinação do percentual de Óleos e Graxas nos tempos inicial, $7 \mathrm{~d}, 15 \mathrm{~d}$ e $21 \mathrm{~d}$ de biorremediação.

\section{Multiplicação dos micro-organismos para a bioaumentação}

Para a produção do biossurfactante utilizaramse cepas de micro-organismos isoladas de efluente rico em lipídios de uma indústria de laticínios, as quais encontravam-se disponíveis no Laboratório de Fermentações do Curso de Engenharia de Alimentos da UPF. Os isolados, mantidos a $4^{\circ} \mathrm{C}$ em meio PCA 
(Plate-Count-Ágar), foram utilizados para o preparo do inóculo, realizado em meio PC (PCA subtraído do Ágar), em erlenmeyers de $250 \mathrm{~mL}$, durante $48 \mathrm{~h}$, a $30^{\circ} \mathrm{C}$. O farelo de soja foi utilizado para veiculação das os isolados ao solo nos processos de bioaumentação atuando como material estrutural e durante o bioaumento por aumento da porosidade do solo. No farelo de soja foi adicionado $5 \%$ de óleo de soja como indutor para a produção de biossurfactantes no meio, esterilizado a $121^{\circ} \mathrm{C}$ durante $20 \mathrm{~min}$ e posteriormente inoculado na razão de $10 \%$ (volume de inoculo/massa de meio). A inoculação foi realizada utilizando-se o inóculo previamente preparado em meio PC. A fermentação do farelo de soja pelos isolados foi realizada a $30^{\circ} \mathrm{C}$ durante $7 \mathrm{~d}$. Foram coletadas amostras nos tempos inicial e final de fermentação para a determinação do índice de emulsificação, a fim de caracterizar a produção de biossurfactantes durante a produção do farelo fermentado para o bioaumento. Os ensaios de bioaumento foram realizados utilizando-se $10 \%$ de farelo fermentado.

\section{Determinações Analíticas}

Determinação do índice de emulsificação nos farelos fermentados

Os biossurfactantes foram extraídos dos farelos fermentados com água destilada a $90{ }^{\circ} \mathrm{C}$ na proporção de $1 \mathrm{~g}$ de farelo para $6 \mathrm{~mL}$ de água a fim de realizar o rompimento da parede celular dos micro-organismos e tornar possível a extração dos biossurfactantes intracelulares. $\mathrm{O}$ prosseguimento da extração foi realizado em banho maria a $160 \mathrm{rpm}$ $(\sim 1,5 \mathrm{~g})$ por $30 \mathrm{~min}$ a $50{ }^{\circ} \mathrm{C}$. Após, foi realizada a centrifugação a $7000 \mathrm{rpm}(\sim 2700 \mathrm{~g})$ por $20 \mathrm{~min}$ e filtração em papel para separação de sólidos e esporos fúngicos, sendo o extrato obtido usado para determinação da atividade de emulsificação.

$O$ índice de emulsificação foi determinado com a finalidade de quantificar o biossurfactante produzido pelos isolados a serem utilizadas na biorremediação. Os testes de emulsificação foram realizados em duplicata e comparados com o controle, sendo misturados $2 \mathrm{~mL}$ de óleo de milho e 3,5 mL de extrato contendo o biossurfactante. Para a realização do branco foi misturado $2 \mathrm{~mL}$ de óleo de milho e 3,5 $\mathrm{mL}$ de água destilada. A mistura foi agitada por $1 \mathrm{~min}$ e após $24 \mathrm{~h}$ de repouso foi realizada leitura da altura da emulsão formada e da altura total da camada de líquido (camada emulsionada + camada não emulsionada) dos tubos, obtendo-se o índice de emulsificação, E24 (BICCA; FLECK; AYUB, 1999). O E24 foi calculado através da Equação 1:

$$
\mathrm{E}_{24}=\frac{\text { altura_da_camada_de_emulsão }}{\text { altura_total }} .100
$$

Determinação da tensão superficial dos extratos de farelo fermentado

O monitoramento da produção de biotensoativos foi realizado medindo-se a tensão superficial dos extratos obtidos dos farelos fermentados, segundo o método do anel de De Nöuy, utilizando-se o tensiômetro Krüss K6.

\section{Determinação de óleos e graxas (O\&G)}

O teor de óleos e graxas (hidrocarbonetos minerais) no solo durante o processo de biorremediação foi quantificado por método de extração contínua em aparelho tipo Soxhlet, segundo a American Public Health Association (APHA, 2000), baseado na quantificação gravimétrica do material extraído com solvente. O método apresentado é adequado para quantificação de lipídeos biológicos e hidrocarbonetos minerais e baseia-se na solubilidade destes constituintes num solvente orgânico (hexano). O percentual de $O \& G$ presentes no solo e o percentual de remoção de óleos e graxas foram calculados pelas Equações 2 e 3, sendo: 
$\mathrm{O} \& \mathrm{G}=$ óleos e graxas;

m: massa;

$\mathrm{RO \& G}=$ remoção de óleos e graxas;

$\mathrm{Cf}=$ percentual de óleos e graxas da amostra no tempo final de biorremediação;

$\mathrm{Ci}=$ percentual de óleos e graxas da amostra no tempo inicial de biorremediação.

$$
\begin{aligned}
& \% O \& G=\frac{m_{\text {óleo }}}{m_{\text {amostra }}} .100 \\
& \% \mathrm{RO} \& G=\left(1-\left(\frac{C_{f}}{C_{i}}\right)\right) x 100
\end{aligned}
$$

\section{Resultados e Discussões}

\section{Remoção de $O \& G$}

A Figura 1 apresenta os gráficos do percentual de O\&G ao longo dos processos de biorremediação (bioaumentação com os isolados L1 e L2 e atenuação natural) do solo contaminado com óleo lubrificante, óleo diesel e óleo de soja, durante $21 \mathrm{~d}$ de experimento. A Tabela 1 apresenta os valores de remoção de O\&G (\%) no tempo de 21 d. A análise de variância dos dados de remoção de óleos e graxas (RO\&G) no tempo de 21 d de biorremediação indicou que houve interação entre as variáveis técnica de biorremediação e contaminante sobre a RO\&G $(p<0,001)$. A comparação das médias dos tratamentos foi realizada pelo teste de Tukey a $5 \%$ de significância (Tab.1).

Verifica-se na Tabela 1 que o óleo de soja foi o contaminante mais facilmente removido do solo independente do processo de biorremediação utilizado. Os percentuais de remoção de O\&G no tempo de $21 \mathrm{~d}$ foram de $87,81 \%, 73,91 \%$ e $49,45 \%$ de remoção de $\mathrm{O} \& \mathrm{G}$, para os processos de bioaumento com os isolados I1 e I2 e atenuação natural, respectivamente. As remoções obtidas para o óleo de soja através dos processos de bioaumentaçao foram significativamente superiores $(p<0,05)$ àquelas obtidas para os demais contaminantes/ técnicas de biorremediação. Isto pode ser explicado pelo óleo de soja ser constituído por estruturas orgânicas simples (ácidos graxos, cuja cadeia contém 8 a 20 átomos de carbono), mais facilmente biodegradáveis que os hidrocarbonetos do óleo lubrificante (mistura complexa de hidrocarbonetos contendo 18 a 40 átomos de carbono e heteroátomos de enxofre, nitrogênio e oxigênio) e do óleo diesel (mistura complexa de centenas de hidrocarbonetos constituídos por 8 a 40 átomos de carbono e hidrogênio e, em menor quantidade, por substâncias cuja fórmula química contém átomos de enxofre, nitrogênio, metais, oxigênio e outros) (SILVA, 2006).

Figura 1 - Óleos e graxas (\%) ao longo do tempo de biorremediação. a) bioaumentação com o isolado 1 (I1); b) bioaumentação com o isolado 2 (I2); c) AN: atenuação natural; OL: óleo lubrificante; OS: óleo de soja; OD: óleo diesel.
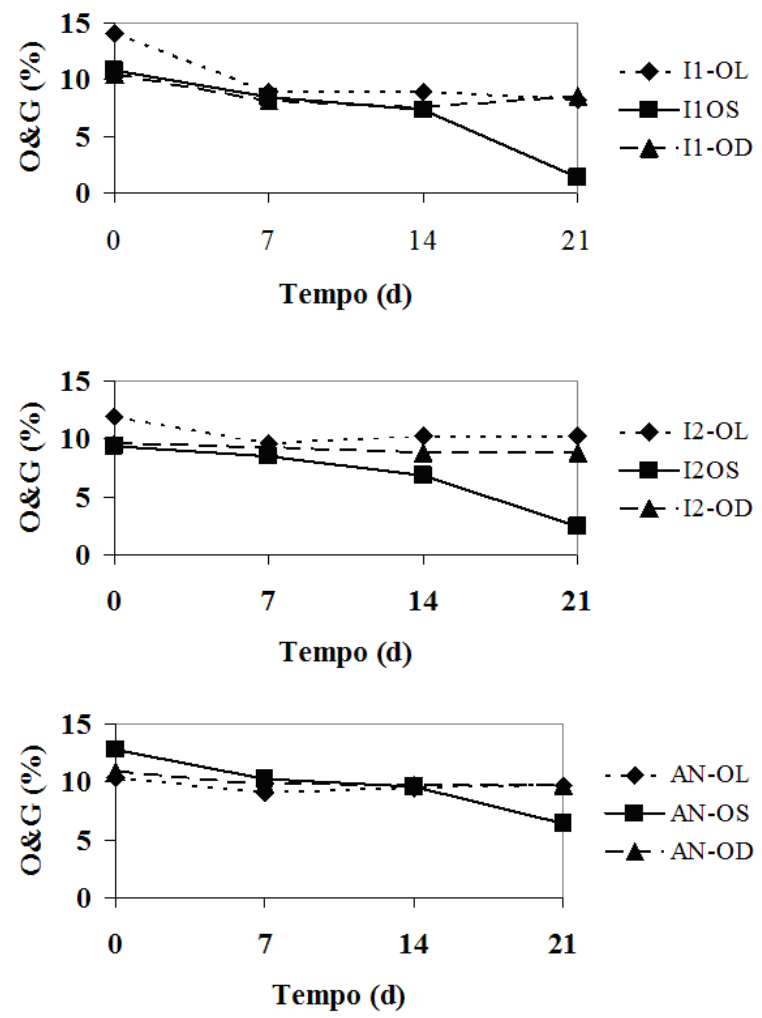

Fonte: Dados do autor 
A biodegradação aeróbia destes compostos envolve o processo respiratório, com oxigênio como aceptor final de elétrons. A biodegradação do óleo de soja é mais facilmente operada pelos micro-organismos aeróbios presentes no solo em comparação com os hidrocarbonetos dos óleos diesel e lubrificante. O óleo de soja é primeiramente hidrolisado por lipases produzidas pelos microorganismos, sendo transformados em glicerol e ácidos graxos. O glicerol pode ser metabolizado via transformação a dihidroxicetona enquanto que os ácidos graxos são convertidos diretamente até acetilCOA pela beta-oxidação para posterior conversão a $\mathrm{CO} 2$ através do ciclo de Krebs (BERG; TYMOCZKO; STRYER, 2006). Em comparação, os hidrocarbonetos de cadeia linear (alcanos, alcenos) primeiramente precisam ser convertidos a alcoóis, aldeídos e posteriormente a ácidos graxos, para então serem convertidos pela beta-oxidação (FRITSCHE; HOFRICHTER, 2005). Já compostos aromáticos como os HPAs e BTEX, presentes no óleo diesel e óleo lubrificante, envolve a conversão do substrato aromático em um metabólito como o catecol, com posterior abertura do anel por enzimas denominadas dioxigenases e conversão a compostos como acetilCoA, oxalato e piruvato, os quais são intermediários do p rocesso respiratório (LEMOS et al., 2009).

Tabela 1 - Remoção de Óleos e Graxas (\%) dos solos contaminados com óleo lubrificante, óleo diesel e óleo de soja no tempo de $21 \mathrm{~d}$ de biorremediação através das técnicas de bioaumentação e atenuação natural

\begin{tabular}{lllc}
\hline Exp & C & TB & $\begin{array}{c}\text { Remoção de O\&G } \\
(\%) *(t=21 \mathrm{~d})\end{array}$ \\
\hline 1 & OL & BI1 & $41,65 \mathrm{~b}$ \\
2 & OL & BI2 & $14,16 \mathrm{c}$ \\
3 & OL & AN & $6,91 \mathrm{c}$ \\
4 & OD & BI1 & $18,54 \mathrm{c}$ \\
5 & OD & BI2 & $7,30 \mathrm{c}$ \\
6 & OD & AN & $11,38 \mathrm{c}$ \\
7 & OS & BI1 & $87,81 \mathrm{a}$ \\
8 & OS & BI2 & $73,91 \mathrm{a}$ \\
9 & OS & AN & $49,45 \mathrm{~b}$ \\
\hline
\end{tabular}

TB: técnica de biorremediação; C: contaminante; OL: óleo lubrificante; OD: óleo diesel; OS: óleo de soja; BL1: bioaumentação com o isolado I1; BL2: bioaumentação com o isolado I2; AN: atenuação natural. *Médias seguidas de letras diferentes indicam diferença significativa em um intervalo de confiança de $95 \%(\mathrm{p}=0,05)$,

O fato da maior remoção de óleo de soja ter ocorrido no tempo de $21 \mathrm{~d}$, pode indicar uma necessidade de adaptação inicial dos microorganismos ao meio contaminado. No processo de atenuação natural, essa tendência é ainda mais evidente para a remoção do óleo de soja, demonstrando que os isolados utilizados nos processos de bioaumentação, em virtude de terem sido isoladas de efluentes ricos em lipídios, podem estar mais adaptadas a este tipo de contaminante que os micro-organismos autóctones do solo.

O percentual médio de remoção de óleos e graxas no solo contaminados com óleo lubrificante no tempo de $21 \mathrm{~d}$ foi de $41,65 \%, 14,16 \%$ e $6,91 \%$ respectivamente, para as técnicas de bioaumento com os isolados I1 e I2 e atenuação natural (Tabelas 1), demonstrando uma maior recalcitrância deste composto em comparação com o óleo de soja. $\mathrm{O}$ bioaumento com o isolado I1 se mostrou mais eficiente $(p<0,05)$ do que a atenuação natural e o bioaumento com o isolado I2 na remoção do óleo lubrificante.

Na biorremediação do óleo diesel os percentuais de remoção de O\&G foram de 18,54\%, 7,30\% e $11,38 \%$, respectivamente, para as técnicas de bioaumento com os isolados I1 e I2 e para a atenuação natural (Tabela 1), sem diferenças significativas entre estes valores de remoção através da comparação de médias pelo teste de Tukey $(p>0,05)$. Os isolados utilizados nos processos de bioaumento não apresentaram maior habilidade para remoção deste contaminante em comparação com os micro-organismos naturalmente presentes no solo. Segundo Barros et al. (2010) esse fato se justifica porque o óleo diesel pode ter influenciado nas condições ambientais e nutricionais dos micro- 
organismos, desfavorecendo assim a remoção desse contaminante no solo. Este comportamento diferiu dos resultados apresentados por Bento et al., (2005), nos quais a bioaumentação foi a técnica que apresentou os melhores resultados em comparação com a atenuação natural e a bioestimulação. $\mathrm{O}$ melhor desempenho para a biorremediação do solo contaminado com diesel foi obtido quando foram adicionados micro-organismos pré-selecionados do próprio ambiente contaminado. Como no presente trabalho os micro-organismos adicionados não foram provenientes do solo, visto a contaminação ter sido simulada e não natural, estas diferenças se justificam.

Comparando-se os micro-organismos utilizados nos ensaios de bioaumentação, verificou-se que o isolado I1 apresentou resultados melhores de remoção de óleos e graxas do que o isolado I2 $(p<0,05)$. Avaliando-se a produção de compostos tensoativos durante a fase de preparo do bioaumento em farelo de soja, verificou-se que os extratos aquosos dos farelos fermentados com os isolados I1 e I2 apresentaram índices de emulsificação de $120 \%$ e $125 \%$, respectivamente, comparados com índices de emulsificação de $15 \%$ observados no tempo inicial de fermentação. Estes compostos podem ter contribuído para a maior remoção do óleo de soja no solo, uma vez que o método utilizado para a determinação da atividade emulsificante usa óleo vegetal como padrão. O biossurfactante produzido pelo isolado I1 durante a produção do farelo fermentado para o bioaumento diminuiu a tensão superficial dos extratos da fermentação de 47,05 $\mathrm{mN} / \mathrm{m}$ (tempo inicial - farelo não fermentado) para $38,5 \mathrm{mN} / \mathrm{m}$, o que pode explicar a maior remoção de óleo de soja obtida para o bioaumento com o isolado I1 (87\%), em comparação com o bioaumento com o isolado I2 (73\%) a qual apresentou diminuição de $47,05 \mathrm{mN} / \mathrm{m}$ (tempo inicial - farelo não fermentado) para 45,1 no seu índice de emulsificação. Segundo Pirolo (2006), é considerado um bom biossurfactante aquele que consegue diminuir a tensão superficial para $40 \mathrm{mN} / \mathrm{m}$ ou menos.

\section{Conclusões}

A bioaumentação apresentou melhores resultados de remoção de óleos e graxas, de 73 a $87 \%$, em comparação com a atenuação natural, de 49\%, para a biorremediação do óleo de soja. O isolado 1 apresentou resultados superiores de remoção de óleos e graxas $(41,6 \%)$ no ensaio de bioaumentação em comparação com o mesmo processo realizado com o isolado $2(14 \%)$ e a atenuação natural $(6,9 \%)$ para o óleo lubrificante. Para a biorremediação do óleo diesel, não houve diferenças significativas entre os processos de bioaumentação e atenuação natural, sendo este o contaminante que apresentou os menores percentuais de remoção (de 7 a 18\%). O maior potencial de remoção de óleos e graxas do isolado 1 pode estar relacionado a produção de biossurfactantes por este micro-organismo.

\section{Referências}

AMERICAN PUBLIC HEALTH ASSOCIATION APHA. Standard methods for the examination of water and wastewater. 20. ed. Washington: APHA, 2000

ANDRADE, J. A.; AUGUSTO, F.; JARDIM, I. C. S. F. Biorremediação de Solos contaminados por Petróleo e seus derivados. Eclética Química, Marilia, v. 35, n. 3, p. 17-43, 2010.

BAPTISTA, S. J.; CAMMAROTA, M. C.; FREIRE, D. D. C. Avaliação da bioestimulação em solos argilosos contaminados com petróleo. In: Congresso Brasileiro de P\&D em Petróleo e Gás, 2., 2003, Rio de Janeiro. Anais... Rio de Janeiro: ABPG, 2003.

BARROS, A. A.; RIZZO, A. C. L.; DA CUNHA, C. D.; SÉRVULO, E. F. C. Monitoramento da atenuação natural de solos artificialmente contaminados com óleo diesel BO e B4. Rio de Janeiro: CETEM, 2010. (Série Tecnologia Ambiental).

BARROS, C. A.; LEMOS, J. L. S. Estudo da degradação de petróleo em solo areno-argiloso com bioaumento fúngico utilizando casca de coco como material estruturante. In: JORNADA DE INICIAÇÃO CIENTÍFICA, 14., 2006, Salvador. Anais... Salvador: CETEM, 2006. 
BENTO, F. M.; CAMARGO, F. A. O.; OKEKE, B. C.; FRANKENBERGER, W. T. Comparative bioremediation of soils contaminated with diesel oil by natural attenuation, biostimulation and bioaugmentation. Bioresource Technology, Essex, v. 96, p. 1049-1055, 2005.

BERG, J. M.; TYMOCZKO, J. L.; STRYER, L. Biochemistry. 6. ed. New York: W.H. Freeman, 2006.

BEZERRA, R. S. Avaliação da adição de PRP no tratamento de solo contaminado por petróleo. In: JORNADA DE INICIAÇÃO CIENTÍFICA, 17., 2009, Ijui. Anais... Ijui: UNIJUI, 2009.

BICCA, F. C.; FLECK, L. C.; AYUB, M. A. Z. Production of biosurfactant by hydrocarbon degrading Rhodococcus rubber and Rhodococcus erythropolis. Revista de Microbiologia, v. 30, n. 3, p. 231-236, 1999.

BUENO, S. M. Bactérias produtoras de biossurfactantes: isolamento, produção, caracterização e comportamento num sistemo modelo. 2008. Tese (Doutorado Engenharia e Ciência de Alimentos) - Programa de Pós Graduação em Engenharia e Ciência de Alimentos. Universidade Estadual Paulista Julio de Mesquisa Filho, São José do Rio Preto.

BUENO, S. M.; SILVA A. N.; GARCIA-CRUZ, C. H. Estudo da produção de biossurfactante em caldo de fermentação. Química Nova, São Paulo, v. 33, n. 7, p. 1572-1577, 2010.

COLLA, L. M.; RIZZARDI, J.; PINTO, M. H.; REINEHR, C. O.; BERTOLIN, T. E.; COSTA, J. A. V. Simultaneous production of lipases and biosurfactants by submerged and solid state bioprocess. Bioresource Technology, Essex, v. 101, p. 8308-8314, 2010.

COSTA, L.; DELLAMATRICE P. M.; DE SOUSA M. V.; SILVA, G. M. M. Biorremediação de uma área contaminada com o inseticida metamidofós por Corynebacterium sp. Conexão Ciência e Tecnologia, Aracati, v. 4, n. 1, p. 17-23, 2010.

DIEMER, F.; SPECHT, L. P.; LAUTENSCHLAGER, C. E.; CONSOLI, N. C. Estudo da permeabilidade do solo da região de Ijuí-RS percolando água e óleo diesel. Teoria e Prática na Engenharia Civil, Rio Grande, n. 16, p. 2941, 2010.

EMPRESA BRASILEIRA DE PESQUISA AGROPECUÁRIA. Centro nacional de pesquisa de solos: sistema brasileiro de classificação de solos. Rio de Janeiro: Embrapa Solos, 2004.

FONTES, G. C.; AMARAL, P. F. F.; COELHO, M. A. Z. Produção de Biossurfactantes por levedura. Química Nova, São Paulo, v. 31, n. 8, p. 2091-2099, 2008.

FRITSCHE, W.; HOFRICHTER, M. Aerobic degradation by microrganisms. In: JÖRDENING, H. J.; WINTER, J. Biotechnology: environmental processes II. Second Edition. Volume 11b, Published Online, 2005.

KANISSERY, R. G.; SIMS, G. K. Biostimulation for the enhanced degradation of herbicides in soil. Applied and Environmental Soil Science, v. 2011, Article ID 843450, 10 pages. DOI:10.1155/2011/843450 v. 2011.

LEMOS, J. L. S.; OLIVEIRA, S. D.; BARROS, C. A.; SCHLITTLER, L. A. F. S. Hidrocarbonetos aromáticos policíclicos (HAPs), propriedades e fatores que afetam sua degradação. Diálogos e Ciência, Salvador, ano 3, n. 11, 79-87, 2009.

LOPES, A.; PIEDADE, M. T. F. O período da contaminação com petróleo influencia a rebrota de Echinochloa polystachya (H.B.K.) Hitchcock em solo de várzea da Amazônia Central? Biota Neotropica, Campinas, v. 10, n. 4, p. 143-148, 2010.

MACIEL, C. C. S.; TAKAKI, G. M. C.; GUSMÂO, N. B. Potencialidade de fungos filamentosos em degradar óleos lubrificantes. Revista Eclesiástica Brasileira, Petrópolis, v. 3, n. 1, p. 58-64, 2010.

MARTINS, V. G.; KALIL, S. J.; COSTA, J. A. V. Lipases and biosurfactant production by solid state fermentation for utilization in bioremediation of vegetable oils and hydrocarbons. Química Nova, São Paulo, v. 31, n. 8, p. 1942-1947, 2008.

MILLER, H. Bioestimulation as a form of bioremediation of soil pollutants. Basic Biotechnology Journal, LOCAL, v. 6 , n. 1, p. 7-12, 2010.

NITSCHKE, M.; PASTORE, G. M. Biossurfactantes: propriedades e Aplicações. Química Nova, São Paulo, v. 25, n. 5, p. 772-776, 2002.

PERELO, L. W. In situ and bioremediation of organic pollutants in aquatic sediments. Journal of Hazardous Materials, Amsterdam, v. 177, n. 1/3, p. 81-89, 2010.

PINTO, M. H.; MARTINS, R. G.; COSTA, J. A. V. Avaliação cinética da produção de biossurfactantes bacterianos. Química Nova, São Paulo, v. 32, n. 8, p. 2104-2108, 2009.

PIROLO, M. P. S. Estudo da produção de biossurfactantes utilizando hidrocarbonetos. 2006. Dissertação (Mestrado em Ciências Biológicas) - Programa de Pós - Graduação em Ciências Biológicas. Universidade Estadual Paulista, Rio Claro.

SILVA, M. V. I. Efeitos do uso do biodiesel sobre propriedades do óleo lubrificante usado em motor de ignição por compressão. 2006. Dissertação (Mestrado em Engenharia Mecânica) - Universidade de São Paulo, São Carlos. 
SOUZA, D. B.; BRITO, G. C. B.; VANCONCELOS, F. C. W.; BRAGA, L. C. Estudo de micro-organismos presentes em uma área contaminada por gasolina comercial. Revista de estudos ambientais, Blumenau, v. 12, n. 2, p. 38-46, 2010.

VIRAMONTES-RAMOS, S.; PORTILLO-RUIZ, M. C.; BALLINAS-CASARRUBIAS, M. L.; TORRES-MUÑOS, J. V.; RIVERA-CHAVIRA, B. E.; NEVÁRES-MOORILLÓN, G. V. Selection of biosurfactan/bioemulsifier-producing bacteria from hydrocarboncontaminated Soil. Brazilian Journal of Microbiology, São Paulo, v. 41, p. 668-675, 2010.

WETLER-TONINI, R. M. C.; REZENDE, C. E.; GRATIVOL, A. D. Degradação e biorremediação de compostos do petróleo por bactérias: revisão. Oecologia Australis, Rio de Janeiro, v. 14, n. 4, p. 1025-1035, 2010.

Recebido em 21 Outubro 2011-Received on October 21, 2011.

Aceito em 16 Janeiro, 2012 - Accepted on January 16, 2012. 
